\title{
Non-invasive estimation of increased LV filling pressures in LV hypertrophy with normal systolic function: Comparison between CMR and Doppler, validated by invasive PCWP measurements
}

\author{
Bernard P Paelinck ${ }^{1 *}$, Paul L Van Herck ${ }^{1}$, Johan M Bosmans ${ }^{1}$, Christiaan J Vrints ${ }^{1}$, Hildo J Lamb ${ }^{2}$ \\ From 2011 SCMR/Euro CMR Joint Scientific Sessions \\ Nice, France. 3-6 February 2011
}

\section{Introduction}

Transmitral flow is unreliable for the estimation of left ventricular (LV) filling pressures in hypertrophy and normal systolic function. Mitral early peak filling velocity $\mathrm{E}$ divided by mitral annulus early peak velocity Ea (E/Ea) and global diastolic strain rate (SR) during peak filling provide a relatively load independent measurement of global myocardial performance.

\section{Purpose}

We aimed to compare Doppler and cardiovascular magnetic resonance (CMR) assessed E/Ea and non-tagged CMR assessed global diastolic SR for the estimation of filling pressure, in comparison with invasive measurement.

\section{Methods}

Sixteen patients with hypertensive heart disease (LV mass index: $111 \pm 18 \mathrm{~g} / \mathrm{m}^{2}$ ), absence of valvular regurgitation and with normal systolic function (LV ejection fraction: $67 \pm 7 \%$ ) referred for cardiac catheterization were studied. Measurement of mitral flow and mitral annulus velocities were performed by Doppler and phase-contrast CMR. CMR derived global longitudinal and global volumetric SR during early peak filling was measured using long-axis cine CMR images. These data were validated by catheter based mean pulmonary capillary wedge pressure (PCWP).

\section{Results}

Mitral flow E/A had no significant correlation with mean PCWP. E/Ea (Doppler $\mathrm{r}=0.74, \mathrm{p}<0.01$ and CMR

${ }^{1}$ University Hospital Antwerp, Edegem, Belgium

Full list of author information is available at the end of the article $\mathrm{r}=0.56, \mathrm{p}<0.05)$, longitudinal diastolic $\mathrm{SR}(\mathrm{r}=0.65$, $\mathrm{p}<0.01)$ and long-axis volumetric diastolic $\mathrm{SR}(\mathrm{r}=0.51$, $\mathrm{p}<0.05)$ related to invasively measured mean PCWP.

Best prediction of elevated mean PCWP was performed by Doppler assessed E/Ea (sensitivity 75\%, specificity: $100 \%$, area under the curve: $0.88, \mathrm{p}<0,05)$. CMR assessed $\mathrm{E} / \mathrm{Ea}$, longitudinal volumetric diastolic SR and long-axis volumetric diastolic SR had similar sensitivity (respectively $75 \%, 87.5 \%$ and $75 \%$ ), specificity (respectively $75 \%, 62.5 \%$ and $87.5 \%$ ) and area under the curve (respectively $0.80,0.80$ and $0.80, \mathrm{p}<0.05$ ) for the prediction of elevated mean PCWP.

\section{Conclusions}

Non-invasive estimation of increased LV filling pressures in LV hypertrophy with normal systolic function can be performed with CMR and Doppler techniques. Doppler assessed E/Ea provided best prediction of elevated LV filling pressure.

\section{Author details}

${ }^{1}$ University Hospital Antwerp, Edegem, Belgium. ${ }^{2}$ Leiden University Medical Center, Leiden, Netherlands.

Published: 2 February 2011

\section{doi:10.1186/1532-429X-13-51-034}

Cite this article as: Paelinck et al.: Non-invasive estimation of increased LV filling pressures in LV hypertrophy with normal systolic function: Comparison between CMR and Doppler, validated by invasive PCWP measurements. Journal of Cardiovascular Magnetic Resonance 201113 (Suppl 1):034. 